\section{Satellite Symposia}

S.S1-1 Satiety, its Determinants, its Assessments

How do we assess satiety: A review of different approaches

France Bellisle

CHRN Ile de France, Bobigny, France

Abstract not received.

S.S1-2 Satiety, its Determinants, its Assessments

Comparison of the satiating power of various high-CHO foods

Marc Fantino

Department of Physiology of Eating Behavior, University of Paris 13, Bobigny, France

Abstract not received.

S.S1-3 Satiety, its Determinants, its Assessments

Satiety: International review of current research and perspectives

John Blundell

INRA, CRNH, Nantes

Abstract not received.

\section{S.S2-1 Carbohydrate Quality and Health \\ Glycaemic response and health - meta-analyses}

Geoffrey Livesey

Independent Nutrition Logic Ltd., Wymondham, Norfolk, UK

Effects on health of "low glycaemic index diets" reported among interventional and epidemiological studies will be shown. In both types of studies there are inconsistent findings, some reporting benefits and some reporting no effects. Inconsistencies (heterogeneity in results) are confirmed by formal meta-analyses. The inconsistencies are partly resolved by modelling of results using meta-regression methods. They are found to relate to a) the range in both the amount and types of carbohydrate ingested and that affect the glycaemic load, and b) to differences among people.

10th European Nutrition Conference
Glycaemic index (GI) provides a means to estimate glycaemic load (GL), but without taking account of the amount of carbohydrate ingested the range of GI people eat fails to explain conflicting observations from epidemiological studies. Moreover, in interventional studies the amounts of glycaemic and non-glycaemic carbohydrate ingested better explains health risk than does GI alone. Limiting glycaemic load (the amount and type of carbohydrate) and limiting i-linear fats (the amount of saturated fats of intermediate chain length and trans fats) are thus each critically important influences on the maintenance of health and management of disease. Information about GI alone is unhelpful.

Factors limiting the glycaemic load in addition to GI are the amount of unavailable carbohydrate and protein in the diet. While fats can also lower GL by replacing carbohydrate, this simply replaces one hazard (glycaemic load) with another (i-linear fats). It is important therefore to keep in mind the selection of foods controlling both GL and i-linear fats.

From interventional studies, there is evidence that significant reductions in GL (more than reductions in GI) reduce food intake, body weight and fasting plasma triacylglycerols. This significant effect is moderate in extent and sufficient to help weight maintenance but insufficient alone for body weight reduction unless shown to persist over the long term. Moreover, it is evident that reduced food intake, body weight and fasting triacylglycerol concentrations are collectively impeded by elevated carbohydrate intake among some taking low GI diets. Avoiding this requires on average a reduction in GL by at least $30 \mathrm{~g}$ glucose eq./d with further reduction evidently providing benefit.

There is also evidence that significant reduction in both the GL and the amount of non-glycaemic carbohydrate (unavailable carbohydrate or fibre, the carbohydrate isomalt) and available carbohydrate that appears as though non-glycaemic will improve blood glucose control. Such improved control appears evident in diabetic patients with respect to fasting blood glucose, and in both diabetics and nondiabetics with respect to glycated haemoglobin and insulin sensitivity. Not all individuals respond equally. In absolute terms diabetics improve fasting blood glucose and glycated protein more than normal healthy people, whereas it seems that insulin sensitivity might improve less in diabetics than in people without diabetes.

Low dose fructose $(\sim 50 \mathrm{~g} / \mathrm{d})$ in place of glycaemic carbohydrates also improves glycated haemoglobin concentrations in both diabetics and non-diabetics, and may (according to the modelling of dose response data) reduce fasting triacylglycerols too. This prediction contrasts with a rise in fasting triacylglycerols seen when nutritionally excessive doses of fructose are consumed.

While these benefits of fructose arise without modification of the energy density of the diet (a factor considered to affect body weight), it cannot be excluded that reduced food intake and body weight found with low GL (or index) diets arises in part from such diets being low energy density, nor that low energy density diets are effective in weight management simply because of both a low GL and a low intake of i-linear fats.

It needs to be considered also that dieting (the deliberate reduction of food intake to achieve a lower body weight) will also lower both the GL and i-linear fat intake.

Considerable interest arises at the present time in health claims. Fat reduction (lowering of i-linear fat content of foods) by replacing fats with carbohydrate simply replaces one hazard with another and has potential to benefit or harm individuals depending on prior diet and choice of carbohydrate foods. i-Linear fat reduction using low or 
non-glycaemic carbohydrate would appear advantageous. Replacement of high glycaemic carbohydrate with low glycaemic or non-glycaemic carbohydrate would appear advantageous too. When choosing fruits, vegetables and whole grain foods (or drinks), forms with a lower GL are expected to be preferable to those with a higher GL. Diets modifications must be though choice of a varied and balanced diet.

\section{S.S2-2 Carbohydrate Quality and Health \\ Indigestible carbohydrates and adipose tissue}

\section{Robertson}

Department of Biomedical and Molecular Sciences, University of Surrey, Guildford UK

In epidemiological studies, insoluble non-viscous dietary carbohydrate have always been shown to be associated with the greatest protection from chronic diseases such as type 2 diabetes, however, the mechanisms have always remained elusive. It is believed now that their documented health benefits are directly related to their fermentation in the human colon and the absorption of short-chain fatty acids (SCFA), the by-products of sacchorolytic bacterial metabolism, into the circulation. Insoluble fibre intake modulates tissue insulin sensitivity in humans, more specifically via a change in adipose tissue metabolism. SCFA appear in the peripheral circulation in micromolar concentrations and using an arterio-venous sampling approach, we were able to demonstrate direct uptake into both fat and muscle tissue. The effects of SCFA had only ever previously been investigated in cell culture, but now we could establish an in vivo effect in humans. Fibre intake suppressed adipose tissue lipolysis, measured by arterio-venous sampling across adipose tissue potentially via a direct effect of these SCFA and recently the appropriate receptors GPR41 and 43 have been identified. As a follow-on to this work in which we were able to modulate the metabolism of subcutaneous adipose tissue, we are currently investigating the effects on other fat stores, specifically visceral fat and ectopic fat depots such as intrahepatic and intramuscular triglyceride stores. It is already established that different fat depots (subcutaneous v visceral) has implications for metabolism and disease risk an additionally when these depots "fail" to buffer the daily fatty acid flux, fat is stored in alternative locations such as the liver, muscle and pancreas with severe implications for insulin sensitivity. In this current study we are investigating the effects of fibre fermentation on body triglyceride distribution in overweight subjects using a combination of MRI and MRS. Preliminary data would indicate that fibre intake has a direct effect of lowering hepatic triglyceride storage, an important site of insulin resistance.
S.S2-3 Carbohydrate Quality and Health

Food process impact on carbohydrate digestibility in cereal bakery wares

\author{
A. Verel \\ Department of Diabetes, University P. et M. Curie, Hôtel- \\ Dieu Hospital, Paris, France
}

Abstract not received.

\section{S.S3 Making Healthier Choices Easy \\ An international, science-based benchmark for front-of-pack labelling}

\section{J. Seide/l}

\section{Choices International Foundation}

Some food products fit better within a healthy diet than others, but how can consumers tell? The 4 to 10 seconds that consumers typically glance at a product prior to purchase is too short to make a judgement on how healthy it is, if consumers have only the nutrition fact box to rely on. A good solution to this is an easily recognisable front-of-pack stamp on healthier choices.

There are two main challenges with this:

- How to distinguish food and beverage products that are healthier options based on sound nutritional science? And how will this fit with the upcoming European nutrient profiles?

- How to ensure a front-of-pack stamp provides consumers with real guidance, motivating them to a behavioural change?

The aim of this symposium is to share knowledge on evaluating individual products' nutritional quality, front-of-pack labelling and the potential impact on product availability and people's health. This shall be done by outlining the European context of promoting healthy diets, including the roles different players can have. Following, the principles and criteria underlying the Choices Programme, as set by an independent scientific committee and globally applicable, will be discussed. The panel discussion will certainly touch upon practical implications, consumer insights, and the fit with existing health strategies.

S.S4-1 Whole Grains and Health: Latest News and Views Whole grains and health - An overview

Joanne Slavin

Department of Food Science and Nutrition, University of Minnesota, 1334 Eckles Avenue, St Paul, MN 55108, US

Abstract not received. 
S.S4-2 Whole Grains and Health: Latest News and Views Whole grains and weight management

Janice Harland; Nicola McKeown

Tufts University, USA

Abstract not received.

\section{Whole grain research in the UK}

S.S4-3 Whole Grains and Health: Latest News and Views

Chris Seal

School of Agriculture, Food \& Rural Development,

University of Newcastle upon Tyne, UK

Abstract not received.

S.S5-1 Almonds; the Heart of the Matter

Diet alternatives to the treatment of cholesterol. Setting the stage, nut and almond epidemiological and clinical trials: 1992 to present

Eric Bruckert

Groupe Hospitalier Pitié-Salpêtrière, Paris, France

Abstract not received.

S.S5-2 Almonds; the Heart of the Matter

Almond consumption and protection against coronary heart disease death in the European Prospective Investigation into Cancer and Nutrition (EPIC)

Joan Sabate

Department of Nutrition, School of Public Health, Loma Linda University, CA 92350, USA

Abstract not received.
S.S5-3 Almonds; the Heart of the Matter

\section{Bodyweight research: Addressing} consumption concerns

James Hollis

Department of Foods and Nutrition, Purdue University, West Lafayette, IN 47906, USA

Abstract not received.

\author{
S.S6-1 Sweertness: Managing Health and Pleasure \\ Psychology and the senses \\ France Bellisle \\ $\mathrm{CRNH}$ lle de France, Bobigny, France
}

Acceptance of sweet stimuli is present at birth in humans, and perhaps even before. Later on, with experience of a certain culture-specific variety of foods, every person develops a unique hierarchy of food likes and dislikes. Sweetness generally remains very much appreciated, although preferred concentrations tend to decrease from childhood to adulthood. The role of a preference for sweet stimuli in obesity has been extensively studied. It appears that overweight is not associated with enhanced liking for sweet tasting products. Obese persons seem to prefer foods that are rich in fats, and foods that are rich in both sugar and fat. Recent studies suggest that intake of sweet carbonated drinks may be associated with risk of weight gain especially in children, although this notion remains controversial. The potential of low-energy intense sweeteners to prevent weight gain or facilitate weight loss seems to be supported by recent reports. Brain imaging studies show that the response of sensitive structures is different after intake of sugar or of intense sweetener solutions. The common idea that sweetness can cause addiction-like behaviours is not supported by any scientific evidence, although the brain mechanisms involved in dependence to substance of abuse could also be involved in the response to high palatability foods (often high in sugar and fat).

S.S6-2 Sweertness: Managing Health and Pleasure Satiety and reward

Adam Drewnowski

Abstract not received.
S.S6-3 Sweertness: Managing Health and Pleasure Symbolism and society

C. Fischler

Centre d'Etudes Transdisciplinaires, CNRS \& Ecole des Hautes Etudes en Sciences Sociales, Paris

Abstract not received. 
S.S7-1 Nutritional Status of Pasta and New Ways of Improvement

Effects of milling and processing on the nutritional properties of pasta products

J. Abecassis

UMR1208 Agropolymer Engineering and Emerging Technologies INRA, SupAgro, UM2, CIRAD, Montpellier, France

Abstract not received.

S.S7-2 Nutritional Status of Pasta and New Ways of Improvement

\section{Low Glycemic Index in pasta products:} Mechanisms and effects

\section{F. Brighenti}

Department of Public Health and Department of Internal Medicine and Biomedical Science, University of Parma, Parma, Italy

Abstract not received.

S.S7-3 Nutritional Status of Pasta and New Ways of Improvement

\section{Nutritional potential of wheat germ:} Quantitative and qualitative aspects

\section{Cara}

Avantage Nutrition, Marseille, France

Abstract not received.

S.S8-1 Public Health Nutrition in the Prevention of Obesity and Metabolic Syndrome

\section{Food habits and public health nutrition}

Lluis Serra-Majem

Department of Clinical Sciences, University of Las Palmas de Gran Canaria, Spain

Abstract not received.
S.S8-2 Public Health Nutrition in the Prevention of Obesity and Metabolic Syndrome

\section{Non exercise activity thermogenesis in prevention of obesity}

James Levine

Endocrine Research Unit, Mayo Clinic and Mayo

Foundation, Rochester, MN, USA

Abstract not received.

S.S8-3 Public Health Nutrition in the Prevention of Obesity and Metabolic Syndrome

\section{Role of industry in the prevention of obesity}

Philips James

London School of Hygiene and Tropical Medicine, United Kingdom

Abstract not received.

S.S9-1 Dietary Fibre, Nutrition and Health: New Scientific Consideration

Dietary fibre and its effects on the
gastrointestinal tract: are all fibres equal?

Martine Champ

INRA, CRNH, Nantes, France

Dietary fibre is still not defined at an international level. If the definition as proposed by AFSSA (2002) is considered, dietary fibre would include carbohydrate polymers of plant origin and processed or synthetic carbohydrate polymers. The present but not validated definition of the Codex Alimentarius would add, to this definition, carbohydrate polymers from animal origin such as galacto-oligosaccharides.

By definition fibres are not digested in the upper part of the digestive tract. They can however exert multiple effects linked to their physicochemical characteristics. As an example viscous fibre such as galactomannan (guar gum) are able to delay gastric emptying and, as a consequence, delay the absorption of most nutrients. Beta-glucans and possibly arabinoxylans from cereals seem to exhibit similar effects.

Most of the fibres (as defined in both definitions) are totally or partly fermented. This fermentation can be fast or slow and occur all along the colon or mostly in its upper part. In most cases, when fast, fermentation produces a large amount of gases which are responsible of flatus in part of the population. Moreover, fast fermentation is also often associated to butyrate production, $\mathrm{pH}$ decrease and prebiotic properties. Most, but not all carbohydrate polymers which exhibit this kind of profile, are non digestible oligosaccharides.

Regarding slowly fermented or partly fermentable polymers or more complex fibre such as intact or poorly damaged cell walls, brans or hulls, most are insoluble. However the profile of short chain fatty 
acids which is produced during this fermentation is highly variable. In that category, there are fractions such as wheat bran which is known to be effective in transit regulation and mainly in constipation.

S.S9-2 Dietary Fibre, Nutrition and Health:

New Scientific Consideration

The impact of fibre on satiety: Implications for weight control?

Margriet Westerterp-Plantenga

Department of Human Biology, Maastricht University, The Netherlands

Abstract not received.

S.S9-3 Dietary Fibre, Nutrition and Health: New Scientific Consideration

\section{Do high fibre diets contribute to a healthy heart?}

Miguel A. Martinez-Gonzalez

Preventive Medicine and Public Health, University of Pamplona, Spain

Abstract not received.

\section{Oral Communications}

\section{OC1-1 Oral Communications \\ A food pattern predicting prospective weight change and its association with risk of cardiovascular disease in the European Prospective Investigation into Cancer and Nutrition (EPIC)-Potsdam study}

D. Drogan; K. Hoffman; M. Schulz; M.M. Bergmann; H. Boeing; C. Weikert

Department of Epidemiology, German Institute of Human Nutrition Potsdam-Rehbruecke, Nuthetal, Germany

Background: Recently, a food pattern has been identified to be predictive for prospective weight change within the EPIC-Potsdam cohort. Given the possible impact of weight change on cardiovascular disease (CVD) risk, we aimed to examine the association between the above mentioned pattern and risk of CVD.

Method: The analyzed food pattern was defined by a high consumption of whole-grain bread, fruits, fruit juices, grain flakes/cereals, and raw vegetables, and a low consumption of processed meat, butter, high-fat cheese, margarine, and meat. The association between quartiles of the food pattern score and CVD morbidity and mortality was examined in 26,256 subjects of the EPIC-Potsdam cohort using a Cox proportional hazards model for competing risks.

Results: During 6.4 years of follow-up, 379 incident cases of CVD were identified of whom 68 were fatal events. The food pattern was not associated with risk of non-fatal CVD. After adjustment for cardiovascular risk factors, the hazard ratios for fatal CVD across increasing quartiles of the score were $1.00,0.85,0.31,0.47$, respectively ( $\mathrm{p}$ for trend 0.016). The association of the food pattern with CVD risk differed between fatal and non-fatal events ( $p$ for difference 0.05 ).

Conclusion: These findings from a large German cohort indicate that a food pattern predicting prospective weight change may be inversely associated with the risk of fatal CVD. 